\title{
Trends in Advanced Imaging Use for Women Undergoing Breast Cancer Surgery
}

\author{
Tara M. Breslin, MD, MS, Mousumi Banerjee, PhD, Cathryn Gust, MS; and Nancy J. Birkmeyer, PhD
}

\begin{abstract}
BACKGROUND: Evidence-based guidelines recommend limited perioperative diagnostic imaging for new breast cancer diagnoses. For patients aged $>65$ years, conventional imaging use (mammography, plain radiographs, and ultrasound) has remained stable, whereas advanced imaging (computed tomography [CT], nuclear medicine scans [positron emission tomography/bone scans], and magnetic resonance imaging [MRI]) use has increased. In this study, the authors evaluated traditional and advanced imaging use among younger patients (aged $\leq 65$ years) undergoing breast cancer surgery. METHODS: The MarketScan Commercial Claims and Encounters Research Database from 2005 through 2008 was analyzed to evaluate the use of conventional and advanced diagnostic imaging associated with surgery for ductal carcinoma in situ (DCIS) or stage I through III invasive breast cancer. RESULTS: The study cohort included 52,202 women (13\% with DCIS and $87 \%$ with stage I-III breast cancer). The proportion of patients undergoing conventional imaging remained stable, whereas the average number of conventional imaging tests per patient increased from 4.21 tests in 2005 to 4.79 tests per patient in $2008(P<.0001)$. For advanced imaging, the proportion of women who underwent imaging increased from $48.8 \%$ in 2005 to $68.8 \%$ in $2008(P<.0001$ ), as did the number of tests per patient (from 1.53 tests in 2005 to 1.98 tests in 2008; $P<.0001$ ). MRI examinations accounted for nearly all of the increase in advanced imaging. Patients who underwent MRI examinations received significantly more traditional imaging tests compared with to those who did not, indicating that these tests are additive and are not replacing traditional imaging. CONCLUSIONS: The current results demonstrate that the use of perioperative breast MRI has increased among women aged $<65$ years. Further study is indicated to determine whether the benefits of this procedure justify increased use. Cancer 2013;119:1251-6. (C) 2012 American Cancer Society.
\end{abstract}

KEYWORDS: breast cancer, imaging, magnetic resonance imaging, patterns of care, technology.

\section{INTRODUCTION}

Cancer care accounts for a substantial and increasing component of medical expenditure in the United States. ${ }^{1}$ Breast cancer, the most commonly diagnosed malignancy among women in the United States, is associated with high initial and ongoing costs compared with other tumor types, and the use of advanced medical technology is a major contributing component to these costs. ${ }^{1,2}$ Data from the Surveillance, Epidemiology, and End Results (SEER)/Medicare linked database demonstrate that, for patients aged $>65$ years, the use of conventional imaging (mammography, plain radiographs, and ultrasound [US]) associated with a breast cancer diagnosis has remained stable, whereas the use of advanced imaging, such as computed tomography (CT) scanning, positron emission tomography (PET) scanning, and magnetic resonance imaging (MRI) has increased. ${ }^{3}$ Although this research describes compelling trends in imaging use for older Americans with breast cancer, 50\% of women who receive treatment for breast cancer are aged $<65$ years at diagnosis. Population-level studies evaluating diagnostic imaging use in younger population are lacking and further study is warranted, because these women may have different preferences and patterns of use for diagnostic imaging. ${ }^{4-6}$

Clinical practice guidelines for breast cancer management recommend limited diagnostic imaging evaluation in conjunction with initial therapy. The objective of traditional diagnostic imaging examinations is to estimate the extent of disease and identify subclinical disease in the affected and contralateral breast to guide local therapy. Advanced imaging technologies such as CT and PET scanning are used to detect metastatic disease, but they are only recommended for patients with higher stage disease (stage IIIA and above). ${ }^{7}$ Breast imaging with MRI may have utility in select situations, such as screening high-risk populations in which a highly sensitive test may be desirable, ${ }^{8}$ but its use for surgical planning in early stage breast cancer is controversial due to its high false-positive rate. ${ }^{6,9}$ To understand patterns of diagnostic imaging use in younger women with breast cancer, we evaluated the use of traditional and advanced imaging for women undergoing breast cancer surgery in a commercial insurance database over a 4-year period.

Corresponding author: Tara M. Breslin, MD, MS, 1500 East Medical Center Drive, 3217 Cancer Center, Ann Arbor, MI 48109-5932; Fax: (734) 936-9647; tarabres@umich.edu

Center for Healthcare Outcomes and Policy, University of Michigan, Ann Arbor, Michigan

DOI: 10.1002/cncr.27838, Received: June 6, 2012; Revised: July 18, 2012; Accepted: July 19, 2012, Published online December 4, 2012 in Wiley Online Library (wileyonlinelibrary.com) 
TABLE 1. Study Cohort Characteristics

\begin{tabular}{|c|c|c|c|c|c|c|}
\hline \multirow[b]{2}{*}{ Characteristic } & \multicolumn{5}{|c|}{ No. of Patients (\%) } & \multirow[b]{2}{*}{$P$} \\
\hline & Totals & 2005 & 2006 & 2007 & 2008 & \\
\hline Total & 52,202 & 9876 & 11,798 & 14,237 & 16,291 & \\
\hline \multicolumn{7}{|l|}{ Age (years) } \\
\hline $18-29$ & 267 & $53(0.5)$ & $60(0.5)$ & $67(0.5)$ & $87(0.5)$ & .9260 \\
\hline $30-39$ & 3435 & $656(6.6)$ & $780(6.6)$ & $927(6.5)$ & $1072(6.5)$ & \\
\hline $40-49$ & 15,463 & 2891 (29.3) & 3521 (29.8) & 4218 (29.6) & $4833(29.6)$ & \\
\hline $50-64$ & 33,037 & $6276(63.5)$ & 7437 (63) & $9025(63.4)$ & $10,299(63.4)$ & \\
\hline \multicolumn{7}{|l|}{ Health plan type } \\
\hline Encounter & 8222 & 1899 (19.2) & 1677 (14.2) & 2033 (14.3) & $2613(14.3)$ & $<.0001$ \\
\hline FFS & 43,980 & 7977 (80.8) & $10,121(85.8)$ & $12,204(85.7)$ & $13,678(85.7)$ & \\
\hline \multicolumn{7}{|l|}{ Disease stage } \\
\hline DCIS & 6271 & 1031 (10.4) & 1355 (11.5) & $1721(12.1)$ & $2164(12.1)$ & $<.0001$ \\
\hline I-III & 45,931 & 8845 (89.6) & $10,443(88.5)$ & $12,516(87.9)$ & 14,127 (87.9) & \\
\hline \multicolumn{7}{|l|}{ Surgical therapy } \\
\hline Lumpectomy & 33,504 & $6530(66.1)$ & 7778 (65.9) & 9015 (63.3) & 10,181 (63.3) & $<.0001$ \\
\hline Mastectomy & 18,698 & 3346 (33.9) & $4020(34.1)$ & $5222(36.7)$ & $6110(36.7)$ & \\
\hline
\end{tabular}

Abbreviations: DCIS, ductal carcinoma in situ; FFS, fee for service.

\section{MATERIALS AND METHODS}

\section{Study Population}

We used the MarketScan Commercial Claims and Encounters Research Database for the years 2005 through 2008. Medstat compiles this database from claims submitted to health plans that contract with large private employers, public agencies, and public organizations in the United States. Health plan types that are included in this database are employer-sponsored, private, fee-for service, and capitated insurance to employees and covered dependents. This longitudinal database tracks all patientlevel inpatient and outpatient claims for as long as the employees remain with their employers.

The study cohort included women ages 18 to 63 years with continuous enrollment in a participating health plan for at least 3 months before and 3 months after the initial diagnosis or surgery date who underwent mastectomy or breast-conserving surgery and had 2 encounters associated with invasive breast cancer (International Classification of Diseases, ninth revision [ICD9] code 174.x) or ductal carcinoma in situ (ICD-9 code 233.0) separated by 30 days. To avoid counting more than 1 episode of care per patient, we analyzed only the first breast cancer-associated episode of care during the study period. ${ }^{10}$ To ensure the most complete data on each patient, women who were aged $\geq 64$ years at the time of initial diagnosis were excluded, because their claims would have been submitted to Medicare when they reached age 65 years, and data from supplemental insurance plans may be incomplete. We also excluded patients who had stage IV breast cancer (ICD-9 codes 197.x, 198.x, and 199.x), because these patients have different indications for imaging compared with patients who have earlier stage breast cancer.

For each individual in the cohort, we extracted data on age, health plan type, stage (DCIS vs stage I-III breast cancer), and claims for breast surgical procedures. Tumor stage was assigned in the following manner: We assumed that patients with invasive breast cancer (ICD-9 code 174.x) without an accompanying code for DCIS (ICD-9 code 233.0) or stage IV breast cancer (ICD-9 codes 197.x, 198.x, 199.x) had stage I through III cancer. Detailed staging information was not available in this data set, so the analysis was performed on the aggregated group. We defined an episode of care to include 3 months before and 3 months after the surgical procedure to capture testing done during the diagnostic and initial treatment phases. Diagnostic procedure codes were used to identify the use of traditional imaging studies, including mammography, plain radiographs, breast US, and advanced imaging studies, including CT, MRI, and PET scanning.

\section{Statistical Analysis}

Univariate tests were used to examine associations between diagnostic testing used, patient age, surgical treatment, and health plan type. Time-trend analysis was used to assess changes in diagnostic testing use over time. Logistic regression analysis was used to model the likelihood of undergoing tests as a function of age, year, and health plan type.

\section{RESULTS}

The initial cohort included 81,878 patients who underwent breast-conserving or mastectomy procedures 

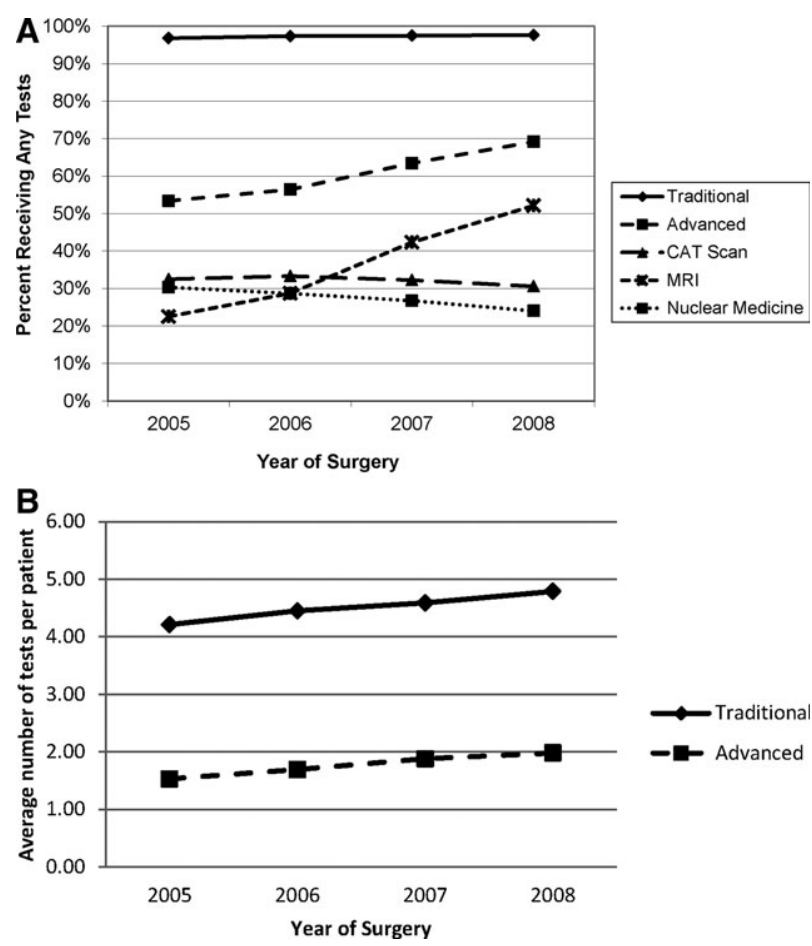

Figure 1. (A) Proportion of patients who underwent advanced imaging in each category. The Mantel-Haenzel chisquare test significant for all test categories $(P<.0001)$ except computed tomography (CAT) scan ( $P=.0011)$; (B) The average number of tests performed per patient in traditional and advanced categories.

between 2005 and 2008. After excluding patients without continuous enrollment for 3 months before and 3 months after the surgery or initial diagnosis date $(n=20,908)$, patients aged $<18$ years or $>64$ years at the time of surgery $(n=288)$, those who had less than 2 claims associated with a breast cancer diagnosis 30 days apart $(\mathrm{n}=8181)$, men $(\mathrm{n}=184)$, and patients with stage IV diagnosis codes or no diagnosis associated with their procedure $(\mathrm{n}=115)$, the final study cohort consisted of 52,202 patients. The number of breast cancer cases identified each year increased, reflecting changes in enrollment by participating plans. The age distribution in the cohort remained stable, whereas enrollment in encounter-based health plans $(P<.001)$, the proportion of patients with stage 0 diagnoses $(P<.001)$, and the use of mastectomy increased modestly $(P<.001)$ (Table 1$)$.

The number of tests per patient associated with a breast cancer episode increased over time in both the conventional and advanced imaging categories. The proportion of women who received at least 1 conventional imaging test was high throughout the study period (97.1\% in 2005 and $97.9 \%$ in 2008); however, the

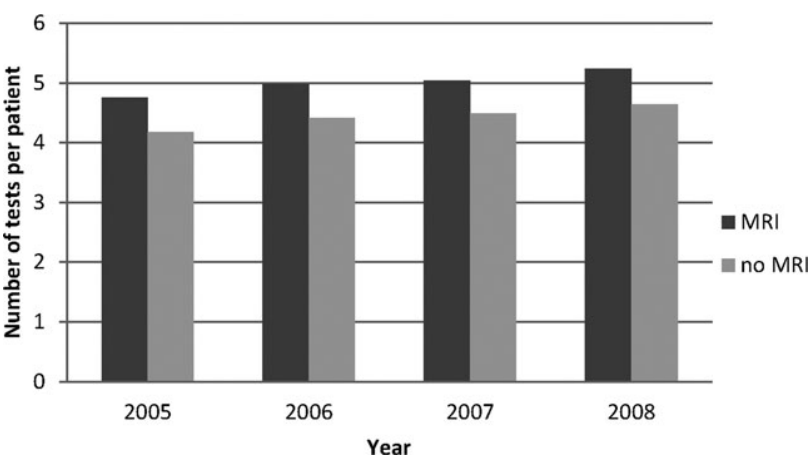

Figure 2. Average number of traditional tests per patient in patients who did or did not undergo magnetic resonance imaging (MRI).

average number of conventional imaging tests per patient increased significantly from 4.21 tests per patient in 2005 to 4.79 tests per patient in $2008(P<.0001)$. The average number of traditional tests per patient was higher in patients who underwent lumpectomy compared with those who underwent mastectomy. Advanced imaging use also increased in terms of both the proportion of patients receiving tests and the number of tests per patient. In $2005,48.8 \%$ of patients underwent at least 1 advanced imaging test. By 2008, this proportion increased to $68.8 \%(P<.0001)$. Similar to traditional imaging, the average number of advanced imaging studies performed per patient increased from 1.53 tests per patient to 1.98 tests per patient in $2008(P<.0001)$ (Fig. 1$)$.

MRI examinations accounted for nearly all of the increase in advanced imaging test use, whereas use of CT and PET scanning declined slightly over the study period. In 2005, 22.8\% of patients underwent an MRI; by 2008, this proportion increased to $52.9 \%$ (Fig. 1, top). Patients who underwent MRI examinations received significantly more traditional imaging tests compared with those who did not (Fig. 2).

The increased use of advanced imaging — and specifically MRI-was observed across all age groups, for both health plan types, and for both DCIS and invasive cancer. The odds of undergoing an MRI were greatest in the youngest age group and for those who had fee-for-service versus encounter-based health plan types, and they increased in each year of the study. After adjustment for age, health plan type, disease stage, and surgical procedure, the odds of undergoing an MRI in 2008 were 3.81 times greater compared with 2005 (Table 2).

\section{DISCUSSION}

In this analysis of claims data from commercial insurance companies, we have documented an increase in use of 
TABLE 2. Odds of Undergoing Magnetic Resonance Imaging Adjusted for Age, Health Plan Type, Stage, Surgical Therapy, and Year of Breast Procedure

\begin{tabular}{ll}
\hline Patient Characteristic & Adjusted OR $(95 \% \mathrm{Cl})$ \\
\hline Age (years) & \\
$50-64$ & $1.52(1.46-1.59)$ \\
$40-49$ vs Ref & $1.79(1.66-1.92)$ \\
$30-39$ vs Ref & $1.91(1.48-2.45)$ \\
$18-29$ vs Ref & \\
Health plan type & $1.10(1.05-1.16)$ \\
FFS vs Encounter & \\
Disease stage & $1.22(1.16-1.30)$ \\
I-III vs DCIS & \\
Surgical therapy & $1.03(0.99-1.07)$ \\
Mastectomy vs lumpectomy & \\
Year of breast surgery & \\
2005 & $1.39(1.30-1.47)$ \\
2006 vs Ref & $2.55(2.41-2.70)$ \\
2007 vs Ref & $3.81(3.60-4.04)$ \\
2008 vs Ref &
\end{tabular}

Abbreviations: $\mathrm{Cl}$, confidence interval; DCIS, ductal carcinoma in situ; FFS, fee for service; OR, odds ratio; Ref, referent category.

both traditional and advanced imaging tests associated with a diagnosis of breast cancer in women aged 18 to 63,2005 to 2008. Diagnostic testing increased in both the conventional and advanced imaging categories, indicating that advanced imaging added to, but did not replace, less expensive traditional imaging in this cohort. Increased use of MRI was responsible for the majority of the increase in advanced imaging use. Although the overall proportion of patients who underwent traditional imaging remained stable, patients who underwent MRI received significantly more traditional tests compared with those who did not undergo MRI.

Our results are consistent with those of other investigators documenting an increase in claims for advanced imaging services in a variety of clinical settings. ${ }^{11-13}$ Several prospective studies have reported increased tumor detection in high-risk women who undergo MRI as part of their breast cancer screening program. ${ }^{8,14}$ MRI is highly sensitive and can identify clinically and mammographically undetectable lesions; for this reason, some in the breast cancer treatment community advocate routine MRI for treatment planning. In support of this concept, single-institution, retrospective reports estimate changes in surgical management for $1 \%$ to $28 \%$ of patients in whom perioperative MRI is used for treatment planning for invasive carcinoma. ${ }^{15}$ However, a recent multicenter, prospective, randomized trial failed to demonstrate an improvement in reoperation rates or health-related quality of life in women who underwent preoperative breast MRI. ${ }^{16}$ In addition, the excellent local control rates that have been achieved using breast-conserving therapy with clinical and mammographic assessment are well established through multiple randomized clinical trials predating the routine use of breast MRI. ${ }^{17}$

There is a lack of agreement in the breast cancer treatment community regarding the precise role of MRI in surgical planning for invasive breast cancer. The National Comprehensive Cancer Network describes MRI testing as an optional procedure for perioperative planning, ${ }^{7}$ whereas others support its use in specific clinical situations. ${ }^{18}$ Still others recommend extreme caution when using MRI for perioperative treatment planning for invasive cancer. ${ }^{9}$ The MRI characteristics of DCIS also have been explored, but there are no evidence-based guidelines recommending the routine use of MRI to guide surgical excision. ${ }^{19}$ Other influences which may have contributed to this increase in MRI use include the increased availability of MRI scanners, ${ }^{20}$ patient demand, institutional pressure to use expensive capital equipment, and potential physician self-referral for diagnostic imaging services. $^{21}$

Our study has several limitations. First, the MarketScan data set contains insurance billing data from select employers, and the patterns of care we describe may not be representative of the care delivered in other settings. Second, administrative billing data do not provide details regarding the specific indications for using various testing procedures or whether or not actual treatment decisions were changed based on test results. Some patients who initially considered breast-conserving surgery may have opted for mastectomy after MRI, and vice versa, depending on test results, personal preference, or physician input. We also did not have access to patientlevel data regarding potential benefits, such as reassurance and diminished anxiety with a negative MRI result, or potential harms, such as increased anxiety and additional testing with a suspicious or positive MRI finding. Third, this data set lacks specific staging information and followup regarding local or distant recurrence, so it is impossible to determine whether the patients who underwent MRI benefited in terms of improved oncologic outcomes. Finally, we did not have access to information regarding whether or not the participating health plans reimbursed for MRI services, so some out-of-pocket MRI use may have been unmeasured.

We have documented a substantial increase in the use of traditional and advanced imaging services 
associated with a breast cancer diagnosis in women aged $<65$ years between 2005 and 2008. This important patient population includes the primary target group for mammographic screening, and $>50 \%$ of incident breast cancer cases occur in this age group. ${ }^{5,22}$ Although the proportion of patients who received traditional imaging remained stable, the number of traditional tests per patient increased. For advanced imaging, both the proportion of patients who underwent tests and the number of tests per patient increased. In addition, patients who underwent MRI received more traditional imaging than those who did not, indicating that MRI did not replace traditional imaging. It is possible that some of the additional traditional imaging tests were done to evaluate abnormalities identified on MRI, indicating that there may be downstream costs associated with MRI testing. We observed an increase in the proportion of patients who were diagnosed with DCIS, which is consistent with national trends. ${ }^{2}$ The increase in use of mastectomy $(P<.0001)$ was statistically significant but numerically small, and we were not able to determine whether this trend reflected increased use of unilateral mastectomy or contralateral risk-reducing mastectomy, as demonstrated in the SEER/Medicare population. ${ }^{23}$

Perioperative MRI is a procedure with uncertain benefits and known monetary costs. The use of this test as well as associated traditional imaging tests is increasing in patients with breast cancer. In an effort to improve patient care and reduce cost, professional organizations have recommended that patients and clinicians question the utility of testing during initial cancer treatment and follow-up. ${ }^{24}$ Although perioperative MRI is not explicitly addressed in these recommendations, clinicians should carefully consider whether using advanced medical imaging at the time of breast cancer diagnosis will provide additional value for their patients or potentially may lead to unnecessary testing and procedures. In addition, additional prospective studies are needed to determine whether patients experience psychological benefit or harm from undergoing MRI and associated testing or have improved outcomes in terms of local or distant disease control.

\section{FUNDING SOURCES}

This study was supported by grant 1R01HS018728-01 from the Agency for Healthcare Research and Quality/National Institutes of Health (Title: "Return on Investment for Quality Improvement Collaboratives in Surgery"; principal investigator, Dr. Birkmeyer).

\section{CONFLICT OF INTEREST DISCLOSURES}

The authors made no disclosures.

\section{REFERENCES}

1. Mariotto AB, Yabroff KR, Shao Y, Feuer EJ, Brown ML. Projections of the cost of cancer care in the United States: 2010-2020. J Natl Cancer Inst. 2011;103:117-128.

2. American Cancer Society. Breast Cancer Facts \& Figures 2011 2012. Atlanta, GA: American Cancer Society; 2011. Available at: http://www.cancer.org/acs/groups/content/@epidemiologysurveilance/ documents/document/acspc-030975.pdf. Accessed November 4, 2011.

3. Dinan MA, Curtis LH, Hammill BG, et al. Changes in the use and costs of diagnostic imaging among Medicare beneficiaries with cancer, 1999-2006. JAMA. 2010;303:1625-1631.

4. Arrington AK, Jarosek SL, Virnig BA, Habermann EB, Tuttle TM. Patient and surgeon characteristics associated with increased use of contralateral prophylactic mastectomy in patients with breast cancer. Ann Surg Oncol.2009;16:2697-2704.

5. DeMichele A, Putt M, Zhang Y, Glick JH, Norman S. Older age predicts a decline in adjuvant chemotherapy recommendations for patients with breast carcinoma: evidence from a tertiary care cohort of chemotherapy-eligible patients. Cancer. 2003;97:21502159.

6. Lazovich D, Raab KK, Gurney JG, Chen H. Knowledge and preference for breast conservation therapy among women without breast cancer. Womens Health Issues. 2000;10:210-216.

7. National Comprehensive Cancer Network (NCCN). Breast Cancer: Clinical Practice Guidelines in Oncology. Fort Washington, PA: NCCN; 2010. Available at: http://www.nccn.org/professionals/ physician_gls/PDF/breast.pdf. [Access date.]

8. Lord SJ, Lei W, Craft P, et al. A systematic review of the effectiveness of magnetic resonance imaging (MRI) as an addition to mammography and ultrasound in screening young women at high risk of breast cancer. Eur J Cancer. 2007;43:1905-1917.

9. Morrow M. Magnetic resonance imaging in the breast cancer patient: curb your enthusiasm. J Clin Oncol. 2008;26:352-353.

10. Azzone V, Frank RG, Pakes JR, Earle CC, Hassett MJ. Behavioral health services for women who have breast cancer. $J$ Clin Oncol. 2009;27:706-712.

11. Korley FK, Pham JC, Kirsch TD. Use of advanced radiology during visits to US emergency departments for injury-related conditions, 1998-2007. JAMA. 2010;304:1465-1471.

12. Smith-Bindman R, Miglioretti DL, Larson EB. Rising use of diagnostic medical imaging in a large integrated health system. Health Aff (Millwood). 2008;27:1491-1502.

13. Mitchell JM. Utilization trends for advanced imaging procedures: evidence from individuals with private insurance coverage in California. Med Care. 2008;46:460-466.

14. Saslow D, Boetes C, Burke W, et al. American Cancer Society guidelines for breast screening with $\mathrm{MRI}$ as an adjunct to mammography. CA Cancer J Clin. 2007;57:75-89.

15. Houssami N, Hayes DF. Review of preoperative magnetic resonance imaging (MRI) in breast cancer: should MRI be performed on all women with newly diagnosed, early stage breast cancer? CA Cancer J Clin. 2009;59:290-302.

16. Turnbull L, Brown S, Harvey I, et al. Comparative effectiveness of MRI in breast cancer (COMICE) trial: a randomised controlled trial. Lancet. 2010;375:563-571.

17. Fisher B, Anderson S, Bryant J, et al. Twenty-year follow-up of a randomized trial comparing total mastectomy, lumpectomy, and lumpectomy plus irradiation for the treatment of invasive breast cancer. N Engl J Med. 2002;347:1233-1241.

18. American Society of Breast Surgeons. Position Statement on the Use of Magnetic Resonance Imaging in Breast Surgical Oncology. Columbia, MD: American Society of Breast Surgeons; 2010. Available at: http://www.breastsurgeons.org/statements/PDF_Statements/ MRI.pdf. [Access date.]

19. Marcotte-Bloch C, Balu-Maestro C, Chamorey E, et al. MRI for the size assessment of pure ductal carcinoma in situ (DCIS): a prospective study of 33 patients. Eur J Radiol. 2011;77:462-467.

20. Baker LC, Atlas SW, Afendulis CC. Expanded use of imaging technology and the challenge of measuring value. Health Aff (Millwood). 2008;27:1467-1478. 
21. Government Accounting Office. Medicare pt B Imaging Services: Rapid Spending Growth and Shift to Physician Offices Indicate Need for CMS to Consider Additional Management Practices. Washington, DC: Government Accounting Office;2008.

22. US Department of Health and Human Services, Centers for Disease Control and Prevention, and National Cancer Institute. National Program of Cancer Registries: 1999-2008 Incidence: WONDER [online database]. Washington, DC: US Department of Health and Human Services, Centers for Disease Control and Prevention, and
National Cancer Institute; 2011. Available at: http://wonder. cdc.gov/cancernpcr-v2008.html. Accessed November 2, 2011.

23. Habermann EB, Abbott A, Parsons HM, Virnig BA, Al-Refaie WB, Tuttle TM. Are mastectomy rates really increasing in the United States? J Clin Oncol. 2010;28:3437-3441.

24. Schnipper LE, Smith TJ, Raghavan D, et al. American Society of Clinical Oncology identifies 5 key opportunities to improve care and reduce costs: the top 5 list for oncology. J Clin Oncol. 2012;30:1715-1724. 